\title{
EDITORIAL
}

\section{Special Section of Neuropsychology Review on HIV/NeuroAIDS}

\author{
Edith V. Sullivan
}

Published online: 26 May 2009

(C) Springer Science + Business Media, LLC 2009

Human immunodeficiency virus (HIV) infection, identified in 1981, expanded rapidly to become a worldwide pandemic, estimated recently to have infected 33 million children and adults worldwide and to result in 2.7 million new infections and 2 million HIV-related deaths annually (UNAIDS 2008 Report on the global AIDS epidemic). Once a sure and early passage to death, HIV infection has become treatable to the point that survival curve analysis predicts the potential of virtually normal longevity with adequate treatment (Hooshyar et al. 2007). Yet, so many variables, such as comorbidity with drug or alcohol abuse, poor nutrition, re-infection, and poor compliance with treatment regimes, can mitigate the benefit of antiretroviral treatments. Consequently, since its recognition, HIV infection has had a variable course, depending on level of infection, medication treatment history, and history of secondary infections and comorbities.

HIV infection is of great relevance to neuropsychologists because of its potential to affect brain function. Through rigorous and longitudinal neuropsychological testing, functional impairments have been identified and linked to affected brain systems. Because HIV-related impairments can be subtle and HIV-infection treatable, impairment detection can require specialized testing and change in status must be considered. Now that HIV-infected individuals have the potential of living a longer, relatively healthy life, it is vital to recognize that treatment progress can be curtailed once again by engaging in risky behavior, such as medication noncompliance, alcohol and drug abuse, and unprotected sex.

This special section of Neuropsychology Review was organized by Robert Heaton, who has been at the forefront of neuropsychological endeavors to identify patterns of sparing and impairment in HIV infected groups and factors modulating these patterns. His selection of authors and topics lends an historical (Cysique and Brew) and world perspective (Roberston, Liner, and Heaton) on the evolution of the infection as affecting the CNS (Ellis, Calero, and Stockin), its relevance to cognitive neuropsychology (Woods, Moore, and Grant), and the influence of neuropsychological impairment on activities of daily life (Gorman, Foley, Ettenhofer, Hinkin, and van Gorp). The effects of comorbidities on neuropsychological functioning are detailed (Martin-Thormeyer and Paul) as are sex differences in expression of neuropsychological deficits and comorbidities affecting the course of HIV infection (Maki and MartinThormeyer). Each paper is rich in its review and provides synthetic considerations for an understanding of the behavioral neuroscience of the disease.

\section{Reference}

Hooshyar, D., Hanson, D. L., Wolfe, M., Selik, R. M., Buskin, S. E., \& McNaghten, A. D. (2007). Trends in perimortal conditions and mortality rates among HIV-infected patients. AIDS, 21(15), 2093-2100.

E. V. Sullivan $(\bowtie)$

Department of Psychiatry \& Behavioral Sciences,

Stanford University School of Medicine,

Stanford, CA 94305-5723, USA

e-mail: edie@stanford.edu 